\title{
Towards Security Risk-oriented Misuse Cases
}

\author{
Inam Soomro and Naved Ahmed \\ Institute of Computer Science, University of Tartu \\ J. Liivi 2, 50409 Tartu, Estonia \\ \{inam,naved\}@ut.ee
}

\begin{abstract}
Security has turn out to be a necessity of information systems (ISs) and information per se. Nevertheless, existing practices report on numerous cases when security aspects were considered only at the end of the development process, thus, missing the systematic security analysis. Misuse case diagrams help identify security concerns at early stages of the IS development. Despite this fundamental advantage, misuse cases tend to be rather imprecise; they do not comply with security risk management strategies, and, thus, could lead to misinterpretation of the security-related concepts. Such limitations could potentially result in poor security solutions. This paper applies a systematic approach to understand how misuse case diagrams could help model organisational assets, potential risks, and security countermeasures to mitigate these risks. The contribution helps understand how misuse cases could deal with security risk management and support reasoning for security requirements and their implementation in the software system.
\end{abstract}

Keywords: Security risk management, Misuse cases, Security engineering, Information system security

\section{$1 \quad$ Introduction}

During the last two decades, line between digital and social life is diminishing, leading that modern society is mainly dependent on information system (IS) and its security. The demand for IS security is constantly growing. Also developing and maintaining system security is increasingly gaining attention. Consideration of IS security at the early stages of software development is also acknowledged in [18]. The security breaches in IS can lead to the negative consequences. The practitioners of IS security must inspect security threats with a negative perspective from the very beginning of IS development process. Consideration of security at early development stages assists to analyse and estimate security measures of the IS to be developed.

This paper discusses the security risk management at requirement elicitation and analysis stage. We will consider the question "how security risk management could be addressed using misuse case diagrams?". To answer this question we analyse misuse cases proposed by Sindre and Opdahl [18]. The misuse case diagrams [17, 18] are one of the possible techniques to relate security analysis and functional requirements of software systems. The main goal is to model negative scenarios with respect to func- 
tional requirements. The misuse cases are already proved to be useful in industry [15]. Existing misuse cases is relatively a simple language, since it contains few constructs to model security concerns. However the previous analysis [9] showed several limitations of misuse cases; for example, misuse cases do not comply with security risk management strategies, because they lack several concrete constructs to address secure assets, security risks and their countermeasures; misuse cases lack distinct constructs for representing security risk concepts These limitations could result in misinterpretation of the security-related concepts leading to poor security solutions. In this paper we tend to propose few improvement to the misuse cases diagrams.

We apply a systematic approach to understand how misuse case diagrams could help to model organisational assets, potential system risks, and security requirements to mitigate these risks. More specifically we introduce new constructs to extend the misuse cases in order to align their constructs with the concepts of Information Systems Security Risk Management (ISSRM) domain model [11, 12]. The benefit of syntactical and semantic extensions is that they introduce the missing semantics in to the language. The domain model is a touchstone to verify if the concepts presented are acceptable and appropriate for the security risk management.

The structure of the paper is organised as follows: in Section 2 we provide background knowledge needed for our study. In Section 3, we describe our research method and introduce Security Risk-oriented Misuse Cases (SROMUC) through an online banking example $[1,8]$. Next we discuss alignment of SROMUC to ISSRM. In Section 4 we review the related work, discuss our results and conclude our study.

\section{Background}

\subsection{Information System Security Risk Management (ISSRM)}

Information System Security Risk Management (ISSRM) [11, 12] is a systematic approach, which addresses the security related issues in an IS domain. The model is defined after a survey of risk management and security related standards, security risk management methods and software engineering frameworks [12]. The domain model (see Fig. 1) supports the alignment of security modelling languages. It improves the IS security and security modelling languages as it conforms to the security risk management of organizations. The model describes three different conceptual categories:

Asset-related concepts describe the organization's assets grouped as business asset and IS asset. It also defines the security criterion as a constraint of a business asset expressed as integrity, confidentiality and availability.

Risk-related concepts define risk, potential harm to business, it is composed of a threat that contains one or more vulnerabilities, if executed successfully, harms the system assets which has negative consequences on assets defined as an impact. They negate the security criterion imposed by the business asset. An event is an abstraction aggregated as a threat and vulnerability where vulnerability is a weakness in a system that can be exploited by threat agent. A threat is a way to inflict an attack. It harms IS and business asset carried out by a threat agent and an attack method to target IS as- 
sets. Threat Agent is an attacker that initiates a threat to harm the IS asset. Attack Method is a mean through which a threat agent executes a threat.

Risk treatment related concepts define a risk treatment decision to avoid, reduce, retain, or transfer the potential risks. It is refined by the security requirement. A control implements the security requirement.

The ISSRM process [11,12] is a 6-step process, based on existing risk analysis methodologies and standards. It starts with context and asset identification of the organization, proceeding to determine the security objectives for identified assets. Next, risk analysis and assessment to examine and estimate potential risks and its impacts. In next step, risk treatment decisions are taken to identify the security requirements. Finally, security control is implemented as security requirement. The process is iterative which may identify new risks and security controls.

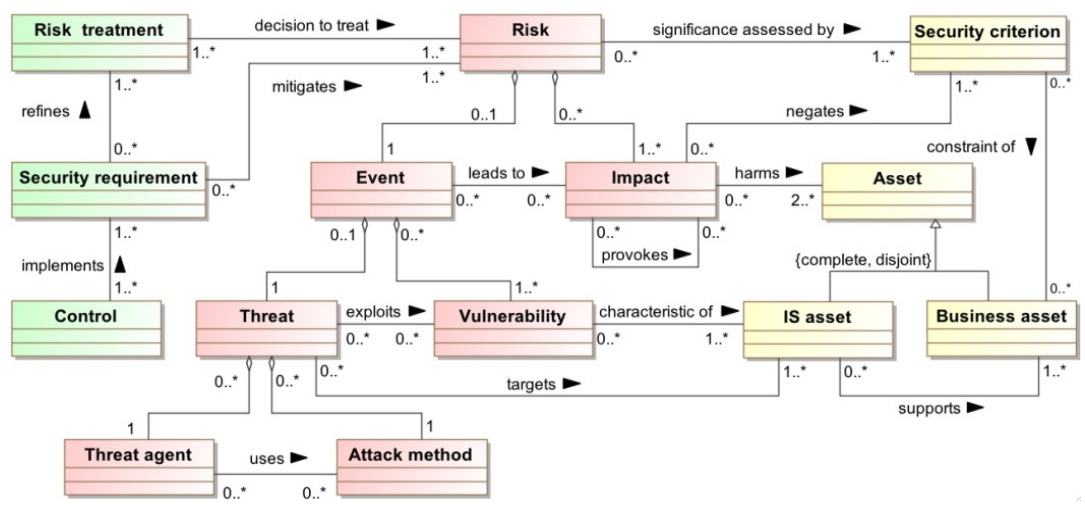

Fig. 1. ISSRM Domain Model [11]

\subsection{Misuse Cases}

Misuse cases are proposed by Sindre and Opdhal in [18]. They have extended the standard UML use cases to model security concerns at the early stages of software system development. The misuse cases include both the graphical notation and textual representation. Sindre and Opdahl define misuse case as a list or sequence of steps, if performed by an agent successfully, cause harm to the stakeholder and/or to the system. They define misuser as an actor that is willing to use the system with unfavourable intents. Initially, only threats were modelled as misuse cases. Later on, Sindre and Opdahl adapted the concept of security use case discussed by Firesmith [6] where security use cases are defined as a function to protect the system assets from the identified risks. In [16] Røstad has extended the misuse cases with a concept of vulnerability as weakness of the system (see a grey-filled use case in Fig. 3).

\section{Security Risk-oriented Misuse Cases (SROMUC)}

This section describes the research method used to develop SROMUC. We illustrate SROMUC using three different security scenarios on asset integrity (see Fig. 2, 3, and 4), confidentiality (see Fig. 5), and availability (see Fig. 6) in an example of online 
banking. This section results in a conceptual alignment between SROMUC and ISSRM domain model.

\subsection{Research Method}

The main research objective of this study is to enable misuse cases to support the security risk management during the IS development. We followed a 3-step research method: firstly, we conduct literature review of security in IS and the ISSRM domain model to identify the security risk concepts. Secondly, we investigate how the misuse case diagrams express the security risk concepts. Hence, we observed the limitations of misuse cases in modelling the ISSRM concepts and executing the risk management process. Lastly, we define misuse case extensions, thus resulting in the Security Riskoriented Misuse Cases (SROMUC). The extensions are done on all three components of the modelling language, namely concrete syntax, meta-model and semantics.

\subsection{Scenario 1: SROMUC Modelling for Integrity}

We illustrate the application of SROMUC using the online banking example $[1,8]$. This scenario is particularly focussed on the IS integrity. To achieve better understandability, we split the scenario to 3 models $^{1}$ : one for assets (see Fig. 2), one for security threats (see Fig. 3), and one for security requirements (see Fig. 4).

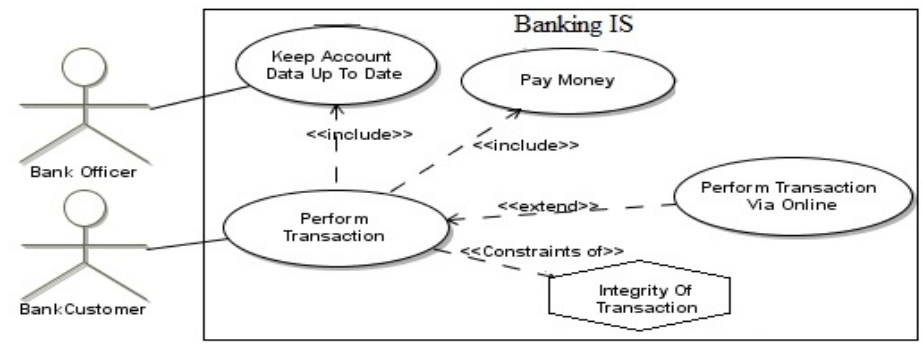

Fig. 2. Asset Modelling

Asset model. In Fig. 2, we illustrate the context of an online banking IS in a use case diagram. A security criterion is a security constraint imposed on business use case (i.e., business asset). The example focusses on the bank customer and bank officer who both communicate with Banking IS. The Bank Customer and Bank Officer are the assets characterising the users of the system in reference to ISSRM domain model. The bank customer seeks to Perform Transaction and bank officer seeks to Keep Account Data Up To Date. The Perform Transaction includes two use cases Pay Money and Keep Account Data Up To Date and extends Perform Transaction Via Online. Perform Transaction has a security criterion Integrity of Transaction represented as a hexagon (see Fig. 2) as it characterises a security constraint of a business use case (i.e., Perform Transaction). In Fig. 2, a dotted line with stereo type

\footnotetext{
${ }^{1}$ To create these models we use the Microsoft Visio tool.
} 
constraints of is linked from business use case (i.e., Perform Transaction) to security criterion (i.e., Integrity of Transaction) shows the relationship between the two. According to ISSRM domain model we identified Perform Transaction as the business asset that has some business value. Hence Perform Transaction Via Online supports the business asset and is considered as an IS asset.

Risk model. In Fig. 3, we model the potential security threat scenario. A misuser (i.e., Attacker) initiates a misuse case (i.e., Intercept Money includes Transfer money to another account and Change details of transaction) by exploiting the vulnerability (i.e., Unsecure Network Channel) in a use case (i.e., IS asset). Following [10] in Fig. 3, this vulnerability is represented by filled grey use case. The misuse case Intercept Payment threatens the use case Perform Transaction Via Online (i.e., IS Asset). The threat Intercept Money leads to an impact (i.e., Money Transferred to Unintended Account) which harms the business use case (i.e., Perform Transaction) and disaffirms the security criterion (i.e., Integrity of Transaction). An impact is a state of system that is represented as rounded rectangle (see Fig. 3). A misuse case is linked to impact using leads to relationship. On one hand, an impact disaffirms the security criterion linked with negates relationship. On another hand impact harms a business use case (i.e., Perform Transaction).

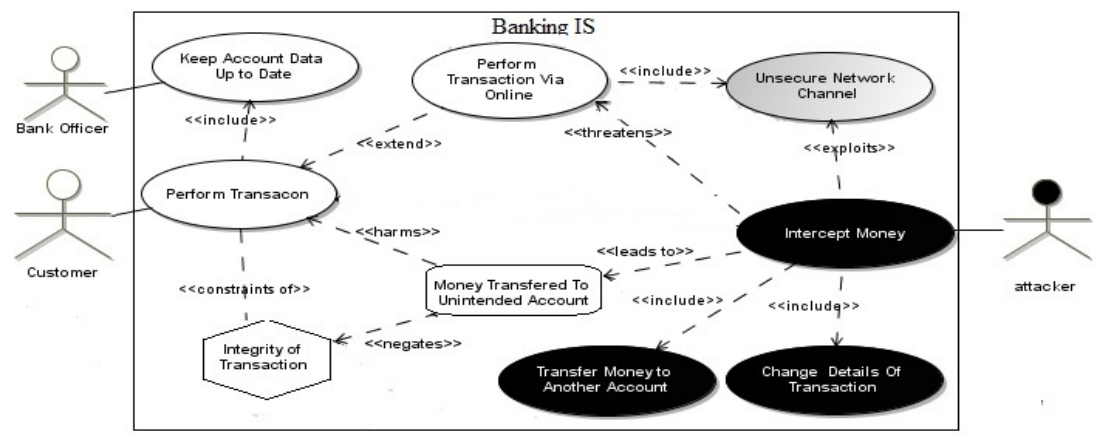

Fig. 3. Threat Modelling

Risk treatment model. The ISSRM domain model defines the risk treatment, control and its implementation. However, SROMUC does not support the modelling of these concepts but security requirement is modelled as a security use case. The security use case is represented as a use case with a lock inside (see Fig. 4). In Fig. 4, we present the security requirement for identified threats. The use case Perform Transaction Via Online (i.e., IS Asset) includes a security use cases (i.e., Apply Cryptographic Procedures and Use Secure Communication Protocol). The security use case mitigates the misuse case (i.e., Intercept Money). It ensures security criterion (i.e., Integrity of Payment) imposed by business use case (i.e., Perform Transaction). 


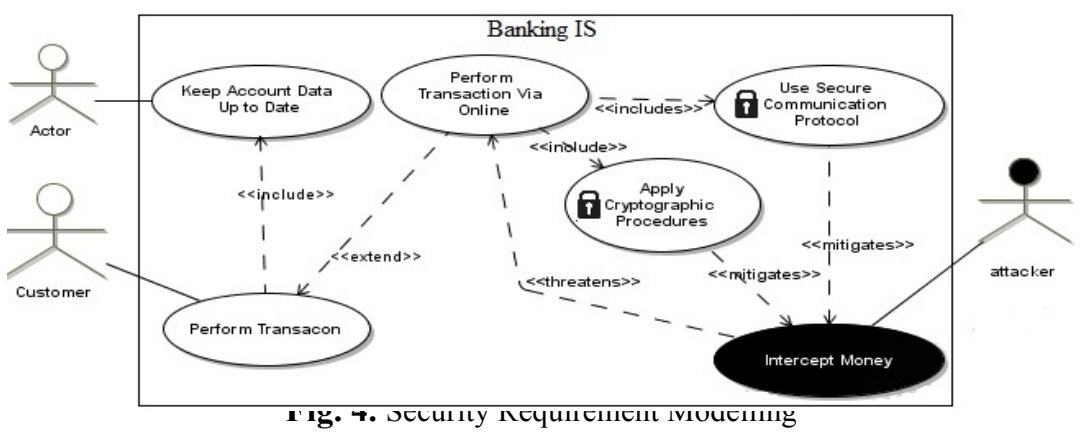

\subsection{Scenario 2: SROMUC Modelling for Availability}

In Fig. 5, we model an online banking IS $[1,8]$ for Availability of Service. In our example, the business use case (i.e., Perform Transaction) has a constraint of security criterion (i.e., Availability Of Online Service). The misuser (i.e., Attacker) initiates a misuse case (i.e., Make Online Service Unavailable includes Initiate Half Opened Connections To Server). It exploits the vulnerability (i.e., Allow Unlimited Number Of Connections) included in a use case Perform Transaction Via Online (i.e., IS Asset). The misuse case Make Online Service Unavailable threatens use case Perform Transaction Via Online (i.e., IS asset) and leads to an impact (i.e., Availability Of Service Is Compromised), moreover, it harms the business use case Perform Transaction. The impact of the misuse case negates the security criterion.

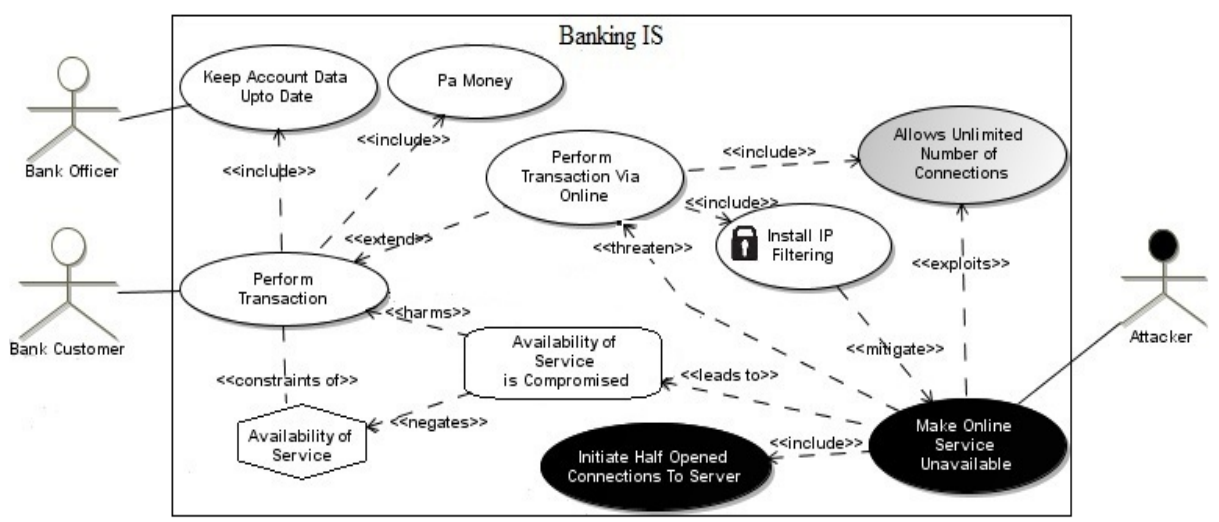

Fig. 5. Modelling for Availability of Service 


\subsection{Scenario 3: SROMUC Modelling for Confidentiality}

In Fig. 6, we model the example of an online banking IS $[1,8]$ for the Confidentiality Of Data. In this example, the business use case (i.e., Perform Transaction) has a constraint of security criterion (i.e., Confidentiality Of Transaction). The use case Perform Transaction Via Online (i.e., IS asset) includes another use case (i.e., Ensure Account privacy includes Enter PIN Code) for securing an online transaction. The misuser (i.e., Attacker) initiates a misuse case (i.e., Steal Account Data includes Retrieve Transaction Data includes Disclose Transaction Data) by exploiting the vulnerability (i.e., Data Is Not Encrypted and Accept Malicious Data). The misuse case (i.e., Steal Account Data) threatens the use case Perform Transaction Via Online (i.e., IS asset) and leads to an impact (i.e., Confidentiality Of Data Is Compromised), moreover, It also harms the business use case (i.e., Perform Transaction). The impact of the misuse case negates the security criterion.

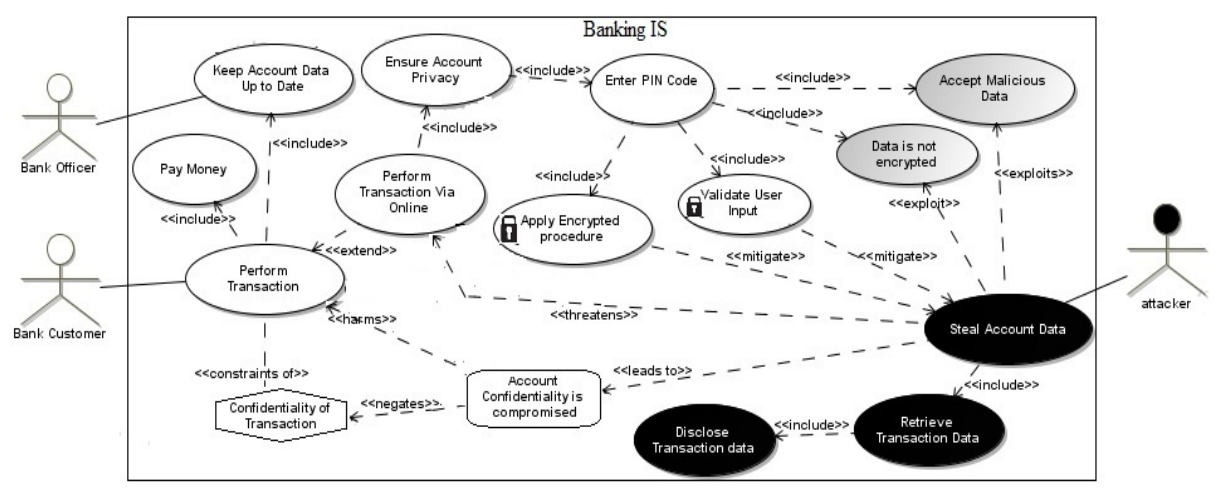

Fig. 6. Modelling for Confidentiality of Data

\subsection{Concept Alignment of SROMUC and ISSRM}

In [9] authors discuss the alignment between the misuse cases and the ISSRM domain model. However it presents only the correspondences, overlaps or/and similarities. In this section we describe the alignment of SROMUC with the concepts found in ISSRM domain model. In Table 1, 2 and 3, first column outlines the ISSRM concepts. The second column expresses their synonyms found in the literature. The third column distinguishes the concepts and relationship. The last column defines the SROMUC visual constructs.

Alignment of asset-related concepts. In Table 1, we introduce SROMUC syntax to represent the ISSRM asset-related concepts. In ISSRM domain model, assets correspond to Actor and Use case in SROMUC. The business asset and the IS asset are modelled as a use case. The supports relationship in ISSRM between IS asset and business assets is expressed using extends and includes relationships. We introduce hexagon construct in SROMUC to represent the ISSRM security criterion. A security criterion is the constraint on business asset therefore the hexagon is linked to business use case through dotted line with constraint of relationship. 
Table 1. Asset Related Concepts (C - Concept, $\mathrm{R}$ - Relationships)

\begin{tabular}{|c|c|c|c|}
\hline ISSRM Concepts & Synonyms & Type & SROMUC Syntax \\
\hline Assets & & $\mathrm{C}$ & 수 $_{\text {actor }}$ \\
\hline Business Asset & Business Use Case & $\mathrm{C}$ & \\
\hline IS Asset & IS Use Case & $\mathrm{C}$ & \\
\hline Security Criterion & Security Constraint & $\mathrm{C}$ & $>$ \\
\hline Supports & - & $\mathrm{R}$ & 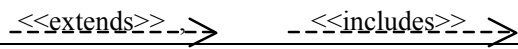 \\
\hline Constraints of & Restriction & $\mathrm{R}$ & $<<$ constraints of $>>$ \\
\hline
\end{tabular}

Alignment of risk-related concepts. In Table 2, we introduce the SROMUC syntax to represent the ISSRM risk-related concepts. In SROMUC, a threat agent is represented as misuser, attack method as misuse case and vulnerability as a use case filled in grey. A threat is modelled as a combination of misuser and misuse case (i.e., misuser communicates with misuse case). The ISSRM targets relationship is represented as an SROMUC threatens relationship. We introduced a rounded rectangle to model the impact concept of ISSRM.

In order to be compliant with ISSRM domain model, we also introduce the exploits, leads to, harms and negates relationships. Exploits relationship defines a link between misuse case and the vulnerability whereas the leads to relationship defines a link between the misuse case and the impact. The harms relationship defines the link between an impact and a business use case whereas a negates relationship defines a link between an impact and the security criterion (see Table 2). We combine the concepts of threat agent, attack method, vulnerability, and impact all together to represent an event, where a risk is understood as a combination of event and the impact.

Alignment of risk treatment-related concepts. In risk treatment-related concepts, we update the visual syntax of security use case by adding a padlock to security use case, which represents security requirement (see Table 3 ). The ISSRM mitigates relationship is modelled with mitigates relationship from security use cases (i.e., security requirement) to misuse case in SROMUC.

Table 2. Alignment of Risk related Concepts(C - Concepts, $\mathrm{R}$ - Relationships)

\begin{tabular}{|l|l|c|c|c|}
\hline ISSRM Concepts & Synonyms & Type & SROMUC Syntax \\
\hline Risk & Hazard & $\mathrm{C}$ & & \\
\hline Impact & & & $\mathrm{C}$ & \\
\hline Event & Effect & $\mathrm{C}$ & \\
\hline Attack Method & Incident & Violence & $\mathrm{C}$ & \\
\hline
\end{tabular}




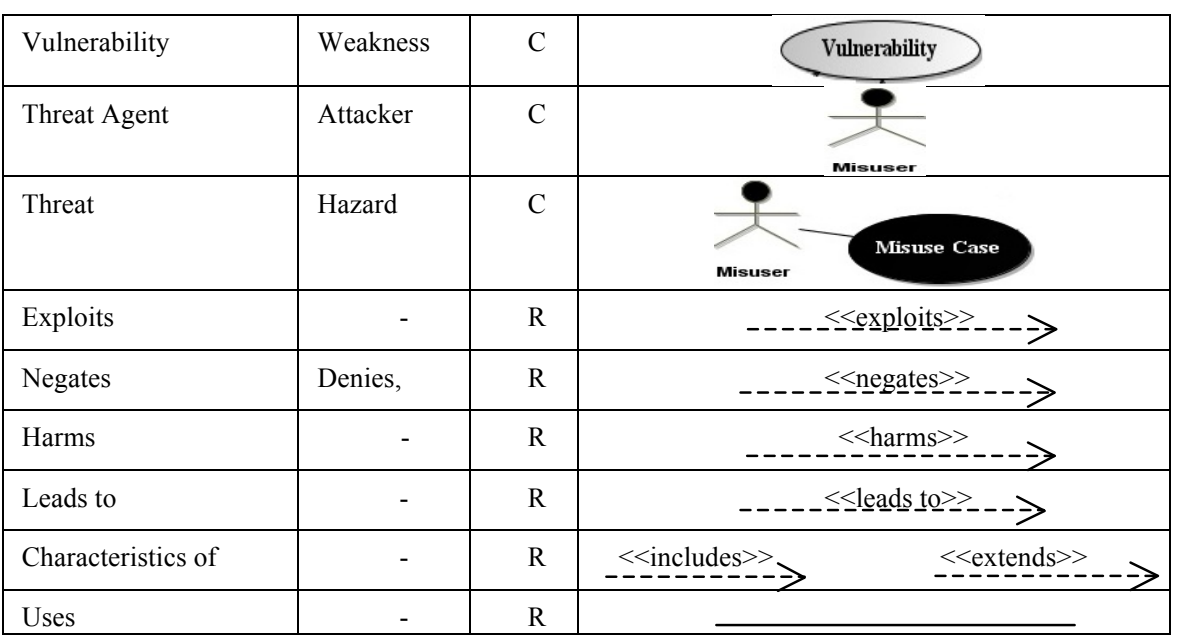

Table 3. Risk Treatment related Concepts (C - Concepts, $\mathrm{R}$ - Relationships)

\begin{tabular}{|c|c|c|c|}
\hline ISSRM Concepts & Synonyms & Type & SROMUC Syntax \\
\hline Risk Treatment & & $\mathrm{C}$ & \\
\hline Security Requirement & Countermeasure & $\mathrm{C}$ & 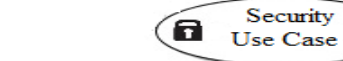 \\
\hline Control & & $\mathrm{C}$ & - \\
\hline Refines & & $\mathrm{R}$ & - \\
\hline Mitigates & Diminishes & $\mathrm{R}$ & - $\leq \leq$ mitigates $>>--->$ \\
\hline Implements & & & - \\
\hline
\end{tabular}

\subsection{Abstract Syntax of Security Risk-oriented Misuse Cases}

In Section 3.1, we presented the SROMUC before abstract syntax due to the simple introduction of the language. However, to illustrate the application of proposed SROMUC, we need to introduce its abstract syntax in Fig. 7. The major elements in the meta-model are an Actor OR Misuser and Use OR Misuse Case. Actor OR Misuser initiates the communication to interact with Use OR Misuse Case. Their cardinality shows that an Actor or Misuser can communicate with one or more Use or Misuser Case. Actor and misuser are the specialisations of an Actor OR Misuser. Use Or Misuse case can includes or extends another Use OR Misuse Case. The Use Case, Vulnerability and Misuse Case are the specialization of Use OR Misuse Case. The Use Case includes one or more Vulnerabilities that can be exploited by one or more misuse cases. A Misuse Case threatens (i.e., threatening) one or more use cases. A Misuse Case Leads To one or more Impact. An Impact Harms one or more use cases (see Fig. 3) by negating one or more Security Criterion define as Constraint Of on that use case. A Security Use Case is a specialised Use Case that Mitigates one or more Misuse Cases. 


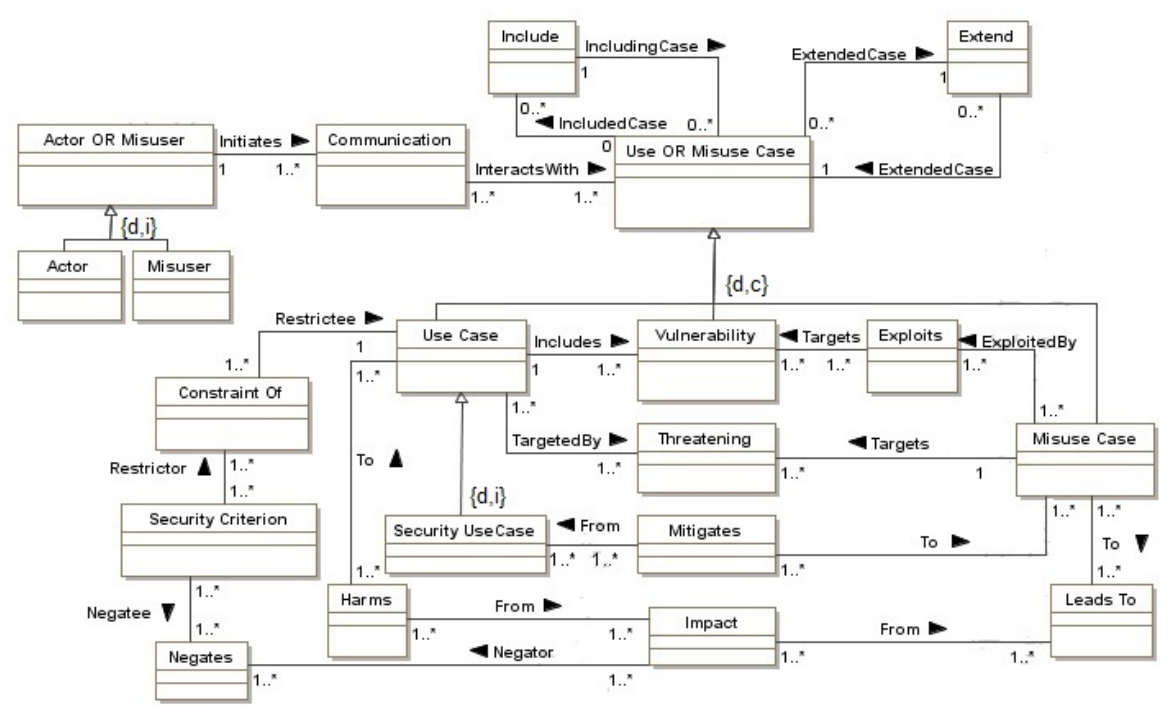

Fig. 7. Meta-model of SROMUC

\section{Discussion and Conclusion}

In this paper, we have analysed how misuse cases can be used to manage security risks at the early stages of the IS development. Firstly, we identified the limitations in existing misuse cases with respect to the ISSRM domain model. Secondly, we extend the language syntax and semantics to respect the ISSRM domain model (see, Tables 1, 2 and 3). This work is a part of the larger effort to align several modelling languages to the ISSRM model that define the semantics at full extend and develop a systematic model transformation-based approach for secure IS development.

\subsection{Related work}

Security Risk Management. The ISSRM covers the identification and specification of security risks, and also supports the risk management process, which focusses on the whole IS, instead of defining security requirements for one or more IS components. The ISSRM approach could potentially be applicable during the IS development while other approaches (see details in [11]) are mainly focused on an existing IS (not its development) and also lacks the Requirement Engineering (RE) activities [11]. In Automated Risk and Utility Management (AURUM) framework [5], when the controls are selected, the decision makers are informed along with the consequences. Whereas, ISSRM integrate the risk management tasks throughout all the stages of IS development. Hence, the risk management tasks and IS development go parallel. Herrmann et al. [7] present a Risk-based Security Requirement, Elicitation and Prioritization (RiskREP) method for managing IT security risks. It defines a set of security requirements, which outline how security as the quality goal can be achieved. 
It performs Business-IT-alignment and prioritises the IT requirement. Similarly, ISSRM align these concepts by supporting the definition of security for the key IS constituents and addresses the IS security risk management process at three different conceptual levels (see Section 2.1).

Misuse cases. There have been few studies carried out on misuse cases and its extension. In $[13,14]$ McDermott and Fox have proposed abuse cases to explore how threats and countermeasures could be modelled using standard UML use case but keeping abuse cases in a separate model. Abuse case focusses on security requirements whereas our approach is aligned with ISSRM and focusses on the overall security risk management. It identifies vulnerabilities and threats, and analyses potential risks and their impacts. Therefore, the elicited security requirements are aligned with the functional system requirements. In [2] Alexander has considered how security use cases can be threatened by misuse cases. Matulevičius et al. [9] have aligned misuse cases with ISSRM however they leave the misuse case extensions for the future development. In this paper the extensions of the misuse cases are built on the previous work of Matulevičius et al. [9] and covers the complete security risk management strategy of an organisation at the early development stage.

\subsection{Discussion}

SROMUC is an approach to elicit security requirements at the early stages of the system development. It will potentially help designers, architects and analysts to understand the potential threats and security attacks. At both the architecture and design stages, risk analysis is a necessity. The SROMUC approach enables the security analysts to discover the architectural flaws so that their mitigation could begin early in the system development. Otherwise disregarding the risk analysis at this level leads to costly problems later. In practice, system stakeholders are not motivated to invest on security concerns, as it does not add direct value to the systems' functionality. The proposed SROMUC strengthens the misuse case diagrams by extending their syntax and semantics. The proposed graphical extensions are not intuitive and they related to the security concerns supported by the ISSRM domain model. However the primary idea is to keep it comprehensible and to compliable with the original definition of (mis)use cases. We differentiate the construct for impact and security criterion from the standard UML use case constructs. The security use case construct has been enhanced to differentiate security requirements from the functional requirements. In [9] Matulevičius et al. have suggested to differentiate the concepts of the IS asset and the business asset. But here, we did not differentiate the assets as it changes the definition of original use case construct. We make an exception regarding the security use because it addresses the system functionality in terms of security countermeasures. Regarding the completeness of alignment between SROMUC and ISSRM domain model, SROMUC does not address the risk treatment and control implementation.

SROMUC is not the only approach that has been aligned to ISSRM domain model. Currently ISSRM is becoming a common model [11] to understand security risk modelling using different modelling languages, like BPMN [3], Secure Tropos [10], 
KAOS extensions to security [11], and Mal-activities [4]. Finally, this may lead to interoperability between different security languages.

Although in the online banking example we have illustrated the applicability and performance of our proposal, we acknowledge the importance of the industrial case study to validate the SROMUC in the practice. As a future work, we also plan to experiment the language in a case study to validate its usefulness and effectiveness.

\section{References}

1. Ahmed, N., Matulevičius, R., Mouratidis, H.: A Model Transformation from Misuse Cases to Secure Tropos. In: Proc of the CAiSE'12 Forum at the 24th International Conference (CAiSE). pp. 7-14. CEUR-WS (2012)

2. Alexander, I.: Misuse cases: Use cases with Hostile Intent. IEEE Soft. 20(1), 58-66 (2003)

3. Altuhhova, O., Matulevičius, R., Ahmed, N.: Towards Definition of Secure Business Processes. In: CAiSE Workshops. vol. 112, pp. 1-15. Springer (2012)

4. Chowdhury, M., Matulevičius, R., Sindre, G., Karpati, P.: Aligning Mal-activity Diagrams and Security Risk Management for Security Requirements Definitions. In: REFSQ, vol. 7195, pp. 132-139. Springer Berlin / Heidelberg (2012)

5. Ekelhart, A., Fenz, S., Neubauer, T.: AURUM: A Framework for Information Security Risk Management. In: HICSS '09. pp. 1-10. IEEE Computer Society (2009)

6. Firesmith, D.: Security Use Cases. Journal of Object Technology 2(3), 53-64 (2003)

7. Herrmann, A., Morali, A., Etalle, S., Wieringa, R.J.: RiskREP: Risk-based Security Requirements Elicitation and Prioritization. In: Perspectives in Business Informatics Research, Riga, Latvia. pp. 155-162. Riga Technical University (2011)

8. van Lamsweerde, A.: Elaborating Security Requirements by Construction of Intentional Anti-Models. In: Proceedings of the 26th International Conference on Software Engineering. pp. 148-157. ICSE '04, IEEE Computer Society (2004)

9. Matulevičius, R., Mayer, N., Heymans, P.: Alignment of Misuse Cases with Security Risk Management. In: Proceedings of 3rd International Conf. on Availability, Reliability and Security. pp. 1397-1404. IEEE Computer Society (2008)

10. Matulevičius, R., Mouratidis, H., Mayer, N., Dubois, E., Heymans, P.: Syntactic and Semantic Extensions to Secure Tropos to Support Security Risk Management. J. UCS 18(6), 816-844 (2012)

11. Mayer, N.: Model-based Management of Information System Security Risk. Ph.D. thesis, University of Namur (2009)

12. Mayer, N., Heymans, P., Matulevičius, R.: Design of a Modelling Language for Information System Security Risk Management. In: Proceedings of the First International Conference on Research Challenges in Information Science, RCIS 2007. pp. 121-132 (2007)

13. McDermott, J.: Abuse-Case-Based Assurance Arguments. In: Proc. of the 17th Annual Comp. Security Applications Conf. pp. 366-. ACSAC '01, IEEE Computer Society (2001)

14. McDermott, J., Fox, C.: Using Abuse Case Models for Security Requirements Analysis. In: Proceedings of ACSAC'99. pp. 55-, IEEE Computer Society (1999)

15. Pauli, J.J., Xu, D.: Trade-off Analysis of Misuse Case-based Secure Software Architectures: A Case Study. In: Proc. of MSVVEIS Workshop. pp. 89-95. INSTICC Press (2005)

16. Røstad, L.: An Extended Misuse Case Notation: Including Vulnerabilities and The Insider Threat. In: Proc. 12th Working Conf. REFSQ'06 (2006)

17. Sindre, G., Opdahl, A. L.: Templates for Misuse Case Description. In: Proc. of the 7th International Workshop on REFSQ'01 (2001)

18. Sindre, G., Opdahl, A. L.: Eliciting Security Requirements with Misuse Cases. Requir. Eng. 10(1), 34-44 (2005) 